\title{
Feature comparison in structural health monitoring of a vehicle crane
}

\author{
J. Kullaa* and T. Heine \\ Helsinki Polytechnic Stadia, P.O. Box 4021, FIN-00099, Helsinki, Finland
}

Received 2007

Revised 2007

\begin{abstract}
Vibration-based structural health monitoring of a vehicle crane was studied. The performance of different features to detect damage was investigated after eliminating the normal operational variations using factor analysis. Using eight accelerometers, ten AR parameters from each record were identified for damage detection. Also transmissibilities between sensors were estimated. Damage was introduced with additional masses at different locations of the structure. All damage cases could be detected from either features using control charts, but transmissibilities proved to be more sensitive to damage than the AR coefficients.
\end{abstract}

Keywords: Structural health monitoring, damage detection, AR model, transmissibility, latent variables, factor analysis, control chart

\section{Introduction}

Structural health monitoring (SHM) is based on damage-sensitive features, the change of which is interpreted as an indication of damage. In vibration-based SHM a few challenges still exist:

1. The features have to be extracted from the measured time histories, which requires system identification.

2. The location or type of damage is generally not known in advance. Therefore, the selection of the monitoring setup is important: type, number, and location of sensors, features to be extracted, etc.

3. Environmental or operational variations can also influence the damage-sensitive features causing false indications of damage. Their effects have to be removed.

4. Monitoring results in a vast amount of data. The steps in damage identification must be automatic.

A widely accepted advantage of vibration-based SHM is that the method is global. This means that damage can be observed remote from the sensor. It can be illustrated with an example that once the stiffness in one part of the structure is decreased, the effect can be observed in the dynamic properties of the structure, for example in natural frequencies and mode shapes.

Several features have been studied for damage identification when the excitation is not known. Some of them are shortly listed in the following, but the list is by no means exhaustive. Natural frequencies and mode shapes have been utilized by several researchers [1]; AR coefficients have been studied e.g. by Sohn et al. [2] and Kullaa [3]; power spectra were investigated by Kullaa [4]; transmissibilities were applied by Worden et al. [5] and Sampaio et al. [6]; wavelets were used by Sun and Chang [7]; and modal filters by Deraemaeker et al. [8].

In this paper, the objective is to study two features used in damage detection: coefficients of the auto-regressive (AR) model and transmissibilities (TR). They are estimated from experimental response data and their performance

\footnotetext{
*Corresponding author. E-mail: jyrki.kullaa@stadia.fi.
} 
to detect damage is compared. The best features are sensitive to damage and result in few false indications of damage. An experimental monitoring test is performed to a vehicle crane by measuring accelerations from eight locations of the structure. The same data records were used to extract both features. Normal operational variability is eliminated from the features before damage detection.

\section{Features}

The two features studied in this paper are the coefficients of the autoregressive (AR) model and the transmissibilities (TR). Both features are related to the spectral properties and are therefore global. The AR model is parametric, while TR is a non-parametric quantity. Therefore, the AR model often results in relatively few features, whereas the number of features in TR can be large. A high number of features can be advantageous, because it is usually not known in advance which features change as the structure is deteriorated. A disadvantage is that the statistical reliability of damage detection decreases with a large number of features (curse of dimensionality) and more training data are needed. AR coefficients are identified from a single sensor, while TR involves a pair of sensors. Independent sensors are technically less demanding, because synchronization between sensors is not needed. However, synchronized sensors provide additional information of the structure, e.g. mode shapes.

\subsection{AR coefficients}

The autoregressive (AR) model of order $n$ is defined as

$$
\begin{aligned}
y(k) & =-a_{1} y(k-1)-\ldots-a_{n} y(k-n)+e(k) \\
& =\varphi^{T}(k) \theta+e(k)
\end{aligned}
$$

$y(k)$ is the response at time $k \Delta t$, where $\Delta t$ is the time increment; $a_{i}$ are the unknown AR coefficients, and $e(k)$ is the error term. The AR coefficients in vector $\theta$ were estimated using the Yule-Walker method [9]. In this study the order $n$ was chosen to be 10 after trying different values and trying to keep the order relatively low.

\subsection{Transmissibilities}

The transmissibility (TR) function is the transfer function between two responses:

$$
\mathrm{TR}_{i j}=\frac{Y_{i}(\omega)}{Y_{j}(\omega)}
$$

where $Y_{i}$ and $Y_{j}$ are the Fourier transforms of responses $y_{i}$ and $y_{j}$ respectively. In practice, TR is computed using the spectral density estimates:

$$
\mathrm{TR}_{i j}=\frac{Y_{i}(\omega)}{Y_{j}(\omega)}=\frac{Y_{i}(\omega) Y_{j}(\omega)}{Y_{j}(\omega) Y_{j}(\omega)}=\frac{S_{i j}(\omega)}{S_{j j}(\omega)}
$$

where $S_{i j}$ is the cross spectral density estimate between $y_{i}$ and $y_{j}$ and $S_{j j}$ is the power spectral density estimate of $y_{j}$. TR is a complex function having information of the amplitude and phase. Transmissibility estimation is easy and fast using the Fast Fourier Transform FFT. Averaging is done in order to decrease the estimation error. In this paper 32 or 64 spectral points are estimated in the frequency range of interest. 


\subsection{Statistic for damage detection}

Statistical methods are used for the damage detection. A control chart [10] is one of the primary techniques of statistical process control. It plots the quality characteristic as a function of the sample number. The chart has lower and upper control limits, which are computed from those samples only when the process is assumed to be in control. When unusual sources of variability are present, sample statistics will plot outside the control limits. In that occasion an alarm signal will be produced. The control chart used in this study is a multivariate Shewhart $T^{2}$, or Hotelling $T^{2}$ control chart [10], where the plotted statistic is:

$$
T^{2}=n(\overline{\mathbf{x}}-\overline{\overline{\mathbf{x}}})^{T} \mathbf{S}^{-1}(\overline{\mathbf{x}}-\overline{\overline{\mathbf{x}}})
$$

where $\mathbf{x}$ is the feature vector, $\overline{\mathbf{x}}$ is the subgroup average (mean of $n$ successive observations), $\overline{\overline{\mathbf{x}}}$ is the process average, which is the mean of the subgroup averages when the process is in control, and $\mathbf{S}$ is the matrix consisting of the grand average of the subgroup variances and covariances. The lower control limit is zero and the upper control limit is

$$
\mathrm{UCL}=\frac{p(m+1)(n-1)}{m n-m-p+1} F_{\alpha, p, m n-m-p+1}
$$

where $p$ is the dimension of the variable, $n$ is the subgroup size, $m$ is the number of subgroups, when the process is assumed to be in control, and $F_{\alpha, p, m n-m-p+1}$ denotes the $\alpha$ percentage point of the $F$ distribution with $p$ and $m n-m-p+1$ degrees of freedom. Its value can be obtained from statistical tables or by using a statistics software. In this study, $n=4, \alpha=0.999$, and the statistic plotted is $T$, the positive square root of $T^{2}$, and the upper control limit is correspondingly scaled. The subgroup size of 4 is a typical value recommended in the statistical process control literature. The $\alpha$ percentage point of the $F$ distribution was chosen very high in order to prevent false alarms. If the data are normally distributed, in the average one sample out of 1000 would fall outside the control limit when the process is in control.

\section{Removing operational variability}

In the vehicle crane, the joint positions, load, friction, or the viscosity of the hydraulic oil can vary during normal operation. Because these operational variations can also influence the damage-sensitive features causing false indications of damage, their effects are first removed using latent variable models. After this data normalization, the effectiveness of different features can be compared.

Factor analysis is a mathematical model that attempts to explain the correlation between a large set of variables in terms of a small number of underlying factors. A major assumption of factor analysis is that these factors are not observed directly. The variables depend linearly upon the factors but are also subject to random errors [11]:

$$
\mathbf{x}=\Lambda \xi+\varepsilon
$$

where $\mathbf{x}$ is a $p \times 1$ vector of the measured variables (features), $\Lambda$ is a $p \times m$ matrix of factor loadings, $\xi$ is an $m \times 1$ vector of unobservable factors, and $\varepsilon$ is a $p \times 1$ vector of unique factors.

In structural health monitoring the measured variables $\mathbf{x}$ can be for example the AR coefficients; the factors $\xi$ are the latent variables affecting those coefficients, e.g. joint positions. The factor model is constructed using the measurements from the undamaged structure under different conditions. This model is then used to estimate the underlying factors and finally to eliminate their effects from the data.

It is assumed that the factors are mutually independent, normally distributed with zero mean and unit variance: $\xi \sim N(\mathbf{0}, \mathbf{I})$. The vector of unique factors $\varepsilon$ is normally distributed with zero means and a diagonal covariance matrix $\Psi: \varepsilon \sim N(\mathbf{0}, \Psi)$. Also, the factors and unique factors are uncorrelated: $E\left(\xi \varepsilon^{T}\right)=0$. The diagonality of $\Psi$ is one of the key assumptions in factor analysis. According to the model (6), $\mathrm{x}$ is therefore distributed with zero mean

$$
\begin{aligned}
E(\mathbf{x}) & =E[(\Lambda \xi+\varepsilon)] \\
& =\Lambda E(\xi)+E(\varepsilon) \\
& =\mathbf{0}
\end{aligned}
$$


and covariance matrix, $\mathbf{R}$ :

$$
\begin{aligned}
\mathbf{R} & =E\left(\mathbf{x x}^{T}\right) \\
& =E\left[(\Lambda \xi+\varepsilon)(\Lambda \xi+\varepsilon)^{T}\right] \\
& =E\left[(\Lambda \xi+\varepsilon)\left(\xi^{T} \Lambda^{T}+\varepsilon^{T}\right)\right] \\
& =E\left(\Lambda \xi \xi^{T} \Lambda^{T}\right)+E\left(\varepsilon \varepsilon^{T}\right) \\
& =\Lambda \Lambda^{T}+\Psi
\end{aligned}
$$

The measured variables are then also normally distributed: $\mathbf{x} \sim N\left(\mathbf{0}, \Lambda \Lambda^{T}+\Psi\right)$.

The objective of factor analysis is to estimate the parameter matrices $\Lambda$ and $\Psi$ that best model the covariance structure of $\mathbf{x}$. This can be done using e.g. the principal factor analysis [11]. The method is iterative regardless of the linearity assumption. From Eq. (8) it can be seen that if $\Psi$ were zero, $\Lambda$ would be obtained using the singular value decomposition (SVD) of the covariance matrix R. Instead, SVD is performed for a modified matrix (from Eq. (8)):

$$
\Lambda \Lambda^{T}=\mathbf{R}-\Psi
$$

However, the estimate of $\Psi$ is not known, and an iterative procedure is needed. Estimates of the factor loadings, $\Lambda$, are obtained by computing the SVD of the $\mathbf{R}-\Psi$ matrix and selecting the $m(m<p)$ largest singular values only. As $\Psi$ is a diagonal matrix, it is obtained by

$$
\Psi=\operatorname{diag}\left(\mathbf{R}-\Lambda \Lambda^{T}\right)
$$

which is substituted in Eq. (9). The procedure is repeated until the estimates of $\Psi$ converge.

Once the model has been identified, the factors $\xi$ and the unique factors $\varepsilon$ must be estimated. The factors can be estimated using the Thomson's factor score [12] which is obtained using the Bayesian approach:

$$
\hat{\xi}=\left(\mathbf{I}+\Lambda^{T} \Psi^{-1} \Lambda\right)^{-1} \Lambda^{T} \Psi^{-1} \mathbf{x}
$$

Finally, the unique factors $\varepsilon$ are computed by

$$
\hat{\varepsilon}=\mathbf{x}-\Lambda \hat{\xi}
$$

which should be independent variables and insensitive to the operational conditions. These unique factors are then used for damage detection. If the structure deteriorates so that the measured variables change, the previously trained factor model cannot explain their changes. These changes would therefore remain in $\varepsilon$ causing an alarm signal in the health monitoring system. The dimensionality of the feature vector (unique factor scores) is not affected by the factor analysis. Therefore, it is usually necessary to perform a dimensionality reduction using the principal component analysis [11] before applying statistical methods to assess damage. The learning phase should contain a full range of operational conditions. It should also be emphasised that the model is solely based on measurement data; no structural model is needed.

\section{Monitoring experiments}

Vibration-based monitoring tests for a vehicle crane HIAB022-2L (Fig. 1) were performed in the laboratory. The features were extracted from the response of the crane vibrating around a slightly varying static equilibrium. The crane was excited with a random excitation using its own hydraulic lift cylinder (Fig. 1). The excitation force was not measured. Eight accelerations (locations and directions shown in Fig. 1) were measured. The first 400 measurements were recorded from the undamaged structure. Fifteen different structural changes were then made using additional masses of five different sizes at three locations. The magnitudes of the additional masses for each damage location were 1.4, 3.4, 5.4, 7.4, and $9.4 \mathrm{~kg}$, labeled as D1, D2, D3, D4, and D5, respectively, and their locations are shown in Fig. 1. Each damage case was monitored with 50 measurements. Each measurement was 60 seconds long and 


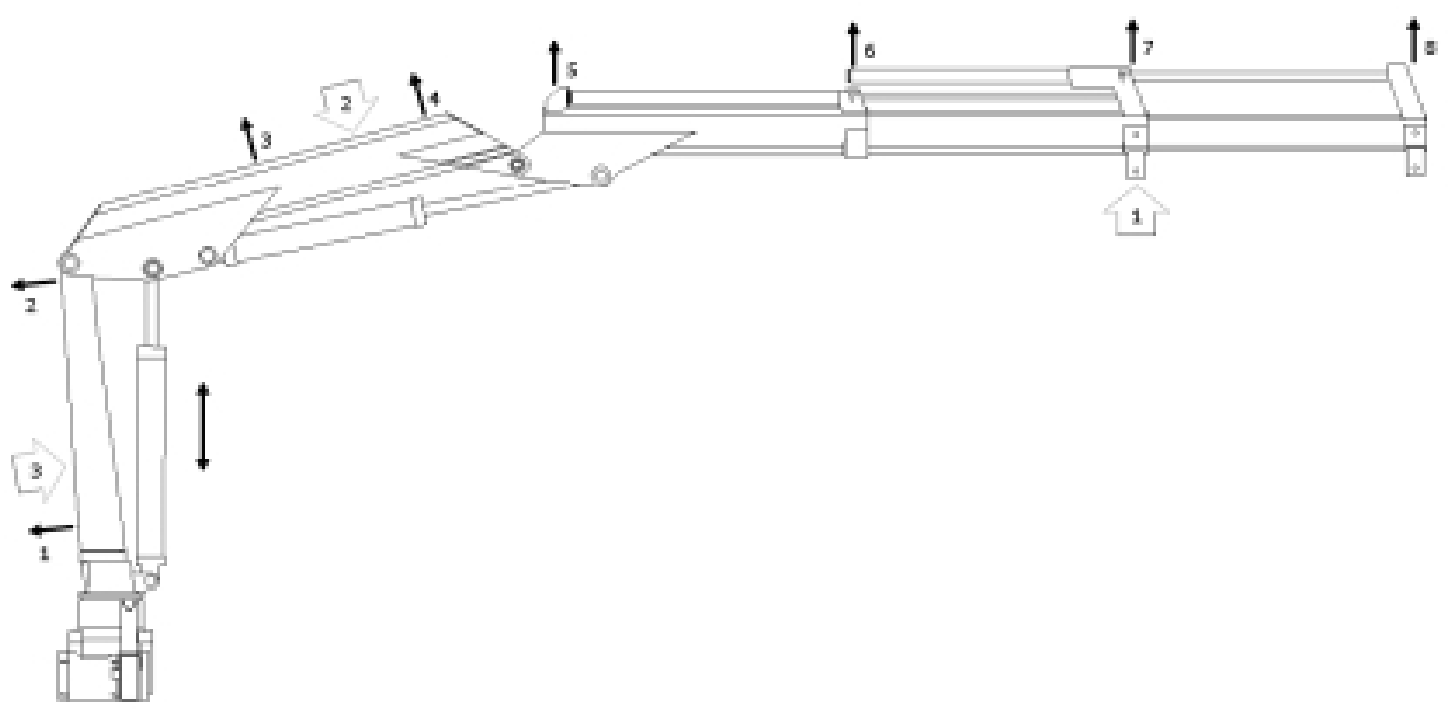

Fig. 1. Vehicle crane HIAB-022-2L. The numbered broad arrows indicate the damage locations, the double arrow is the excitation, and the single arrows are the response locations.
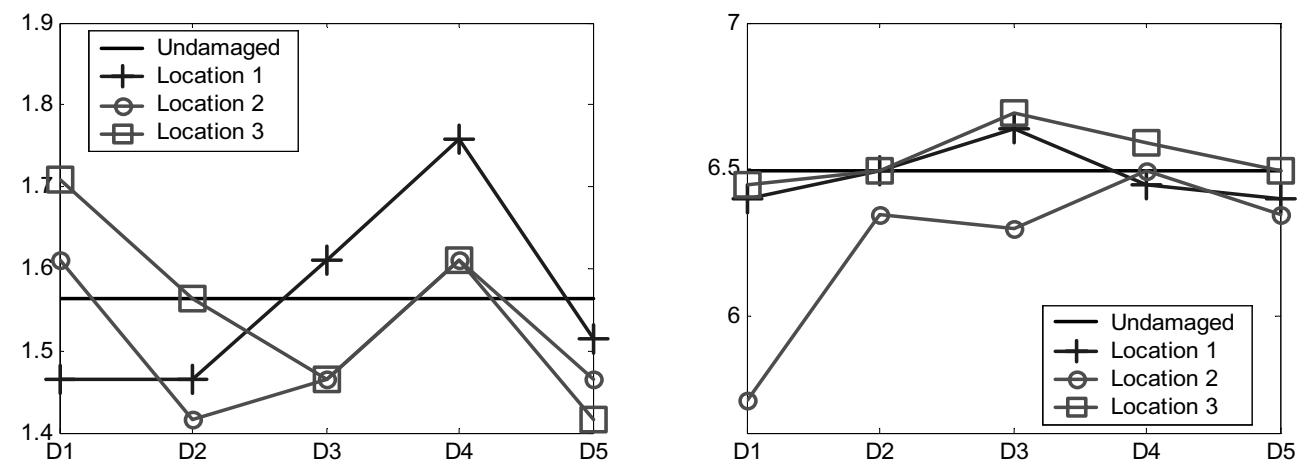

Fig. 2. Natural frequency variation due to added masses. Left: $1^{\text {st }}$ frequency; right: $2^{\text {nd }}$ frequency

filtered with a $100 \mathrm{~Hz}$ low-pass filter. The tests were performed during three days, a relatively short time period. Much later, control measurements were made without additional masses to check if the system had changed.

In order to see the effect of the added mass, the two lowest natural frequencies were identified from a randomly chosen sample at each damage location and level. The frequencies are shown in Fig. 2. The horizontal line is the undamaged reference frequency. The mass increase should decrease the natural frequencies, but in some cases the frequencies were higher than the reference value, showing the fact that the mass effect was small compared to that of the operational variability.

Ten AR coefficients were extracted from each time record resulting in 80 features from which five are plotted in Fig. 3 left including the undamaged structure (samples 1-400), damage location 1 (samples 401-650), and the control measurements (samples 651-777). Transmissibilities were estimated between sensor pairs 1-2, 3-4, 5-6, 5-7, and 5-8 (Fig. 1). These pairs were chosen to see if it is easier to detect damage in a boom from the transmissibilities between the sensors on that boom, or if the transmissibilities give global information of the structure. The absolute transmissibilities using FFT length of $N F F T=32$ resulted in estimation at $N F F T / 2+1=17$ frequencies for each sensor pair. The total number of features was then 85 . Five selected features from the undamaged structure and damage location 1 are shown in Fig. 3 right. 

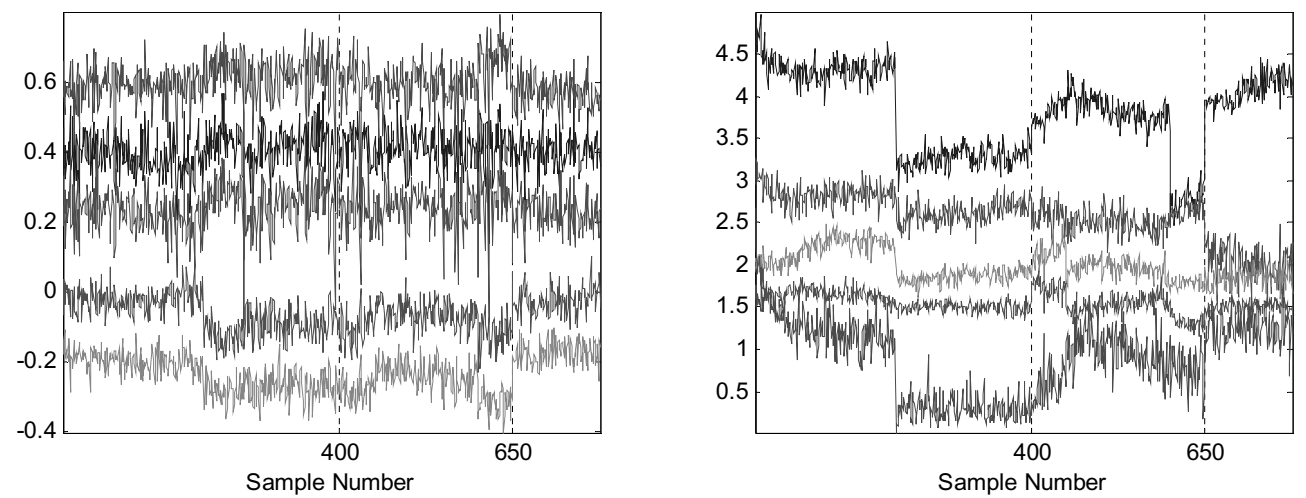

Fig. 3. Five selected AR coefficients (left) and absolute transmissibilities (right). Damage location 1.
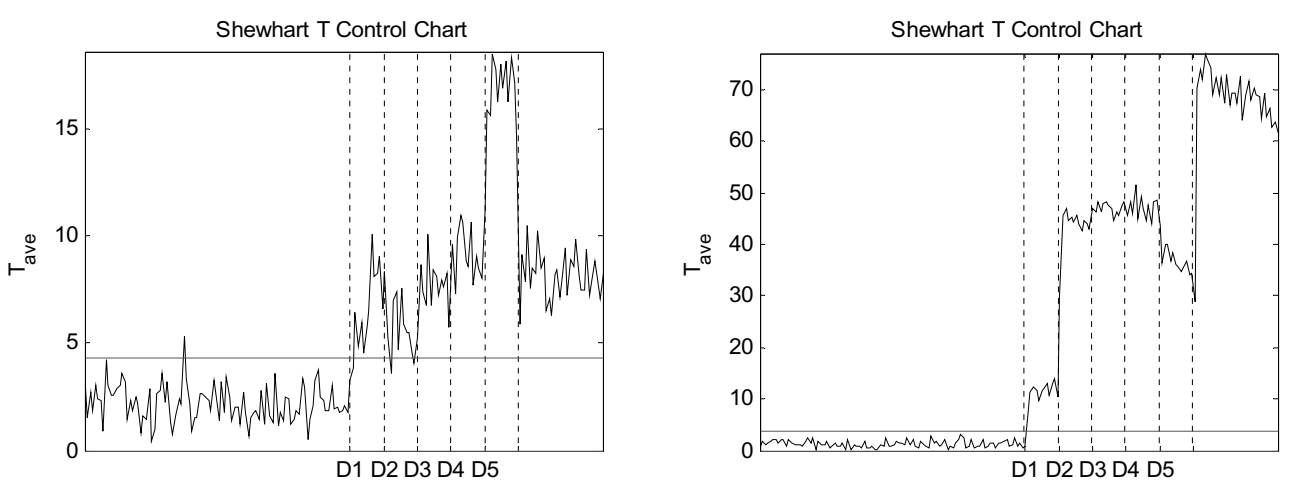

Fig. 4. Control charts for AR coefficients (left) and absolute transmissibilities (right). Damage location 1.

From Fig. 3 it can be seen that the variation of the features was large, especially with the transmissibilities. Also, clear jumps in features can be observed at certain occasions. This is due to applied lubrication to joints and larger motions driven with the crane between measurements. In particular, the effect after sample 196 is clearly seen, while the crane is known to be healthy. Damage detection was performed using control charts [10]. Every other sample between 1 and 400 were used as training data and samples 1-300 were used as the in-control samples to compute the control limits for the control chart. Control charts for the features after factor analysis and subsequent principal component analysis are shown in Fig. 4. Each statistic is the average of four successive features and the damage is consequently introduced at sample number 100. It can be seen that damage was detected with either features, but the control data signalled as well. This is an indication of a change in the system, a change that is not exactly known. It can be also due to a relatively small amount of training data or change in the operational conditions. Recall that the control measurements were performed much later than the actual monitoring experiments. It can be concluded that in practice the collection of the training data should be scheduled for several days. To see how the damage (added masses) can be observed with the proposed features, in the subsequent analyses the training data are extended to have also every second sample of the control data.

To see the effect of the factor analysis to remove the operational variability, the AR coefficients for damage location 1 were analysed with and without factor analysis. The control charts are plotted in Fig. 5. The advantageous effect of the factor analysis is obvious. The operational influences could be eliminated from the data, even without knowing the affecting quantities. Factor analysis is applied to all subsequent analyses.

The performance of the two features to detect the three damage locations is studied in the following. Control charts for damage locations 1,2, and 3 are shown in Figs 6, 7, and 8, respectively. The analyses of the AR coefficients are shown in the left, and transmissibilities in the right. All damage cases were correctly detected from both features and without false indications of damage. For damage location 1, the damage size evolution can also be observed, 

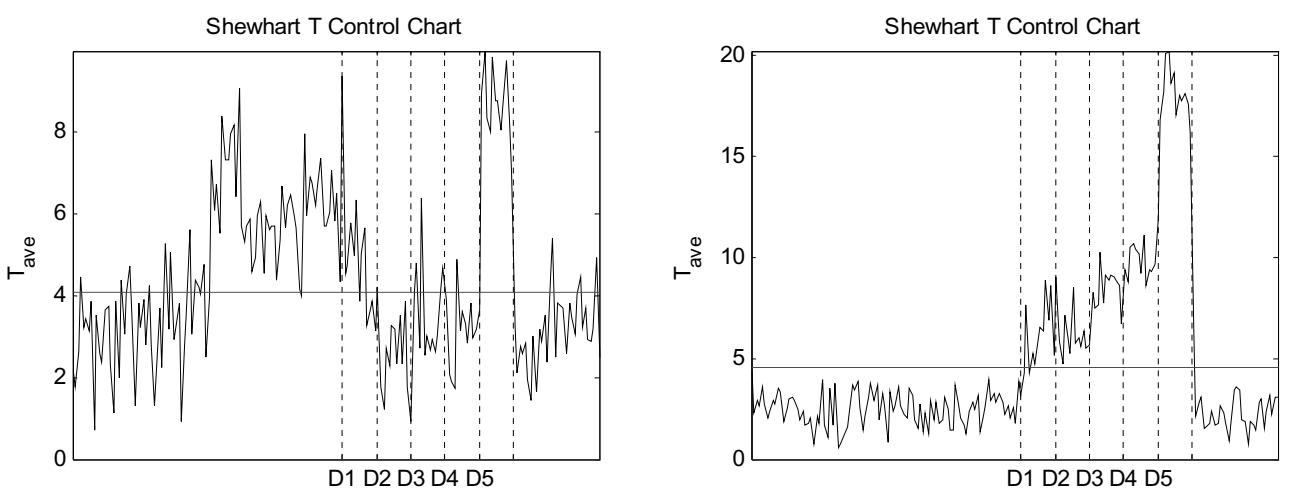

Fig. 5. Control charts of the largest PC:s of the AR coefficients with damage location 1. Left: without FA; right: with FA.
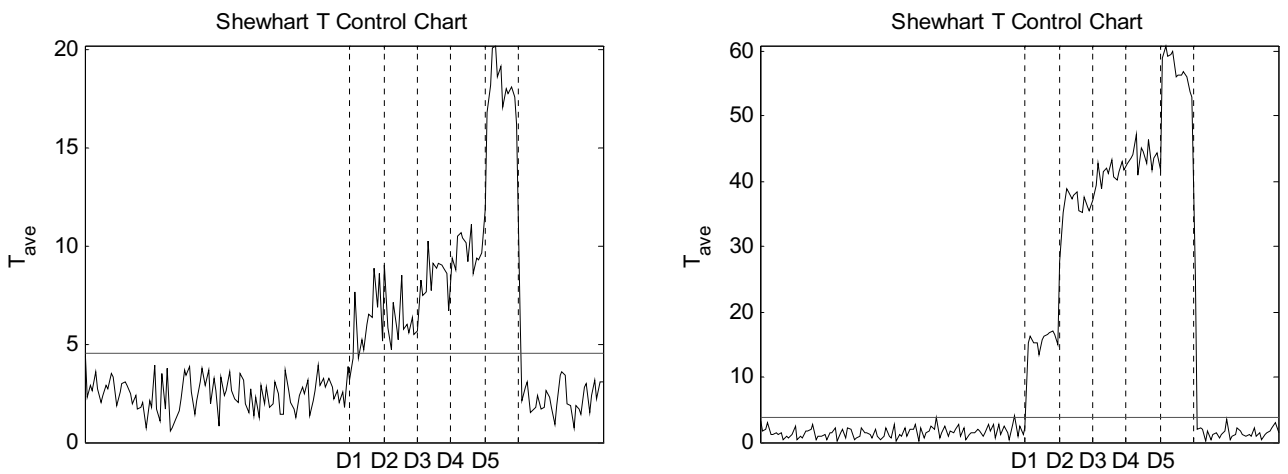

Fig. 6. Damage location 1. Left: AR coefficients. Right: absolute transmissibilities.
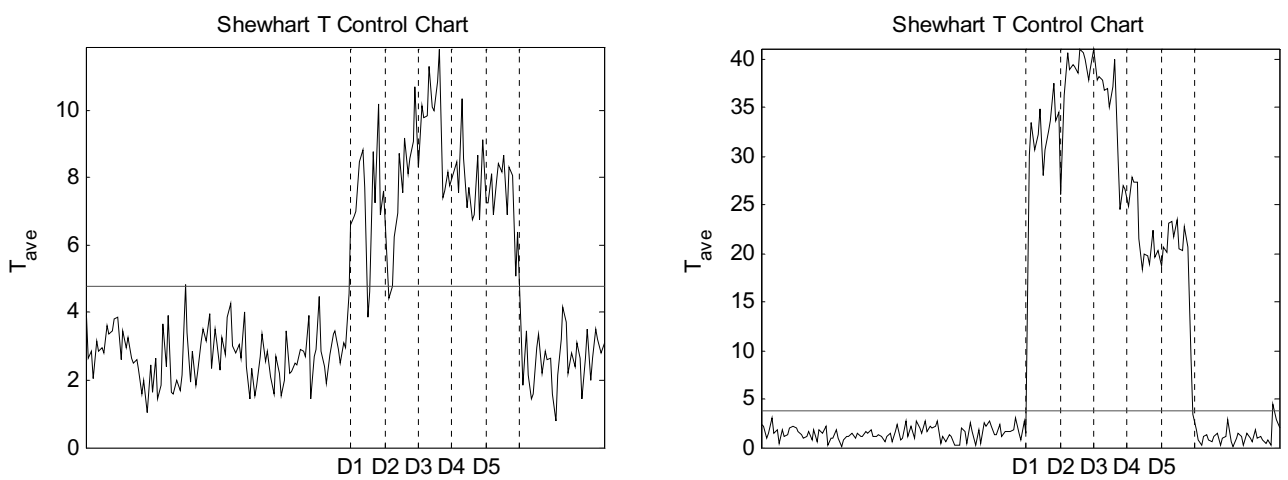

Fig. 7. Damage location 2. Left: AR coefficients. Right: absolute transmissibilities.

whereas for damage locations 2 and 3 the damage size is not clearly seen from the control charts. This is probably due to different operational conditions not present in the training data, the effect of which could not be removed. Transmissibilities proved to be more sensitive to damage than the AR coefficients. Transmissibilities showed no local effectiveness (not shown here). All damage cases were detected from transmissibilities both near damage and remote from damage.

The most sensitive results were obtained using complex transmissibilities (Fig. 9). Transmissibilities having 32 and 64 spectral lines were estimated, resulting in 160 and 320 features, respectively. It should be noted that the training data consisted only 264 samples, which was less than the dimensionality of the feature vector. Using 

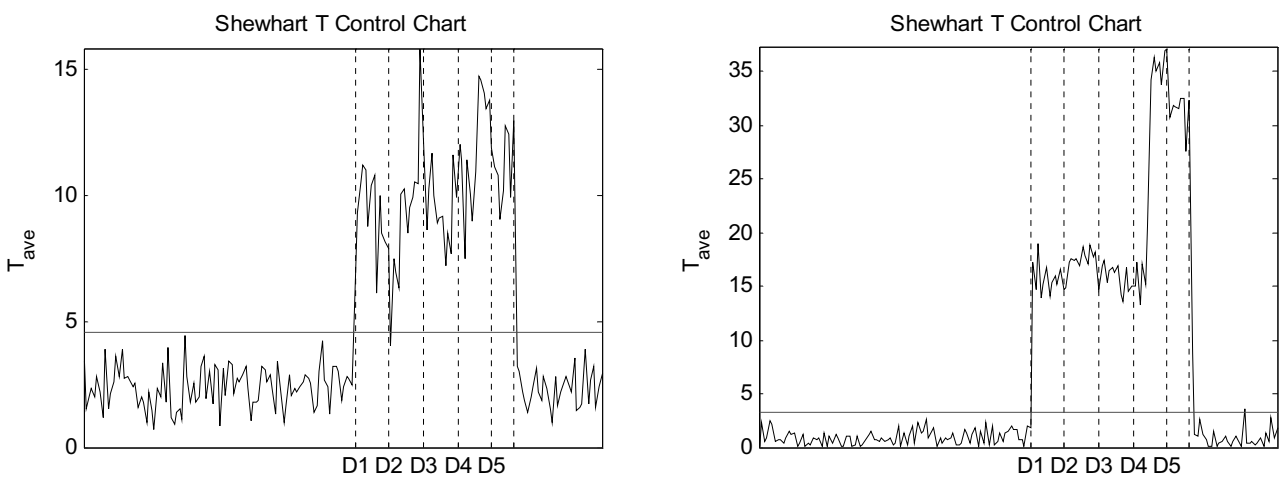

Fig. 8. Damage location 3. Left: AR coefficients. Right: absolute transmissibilities.
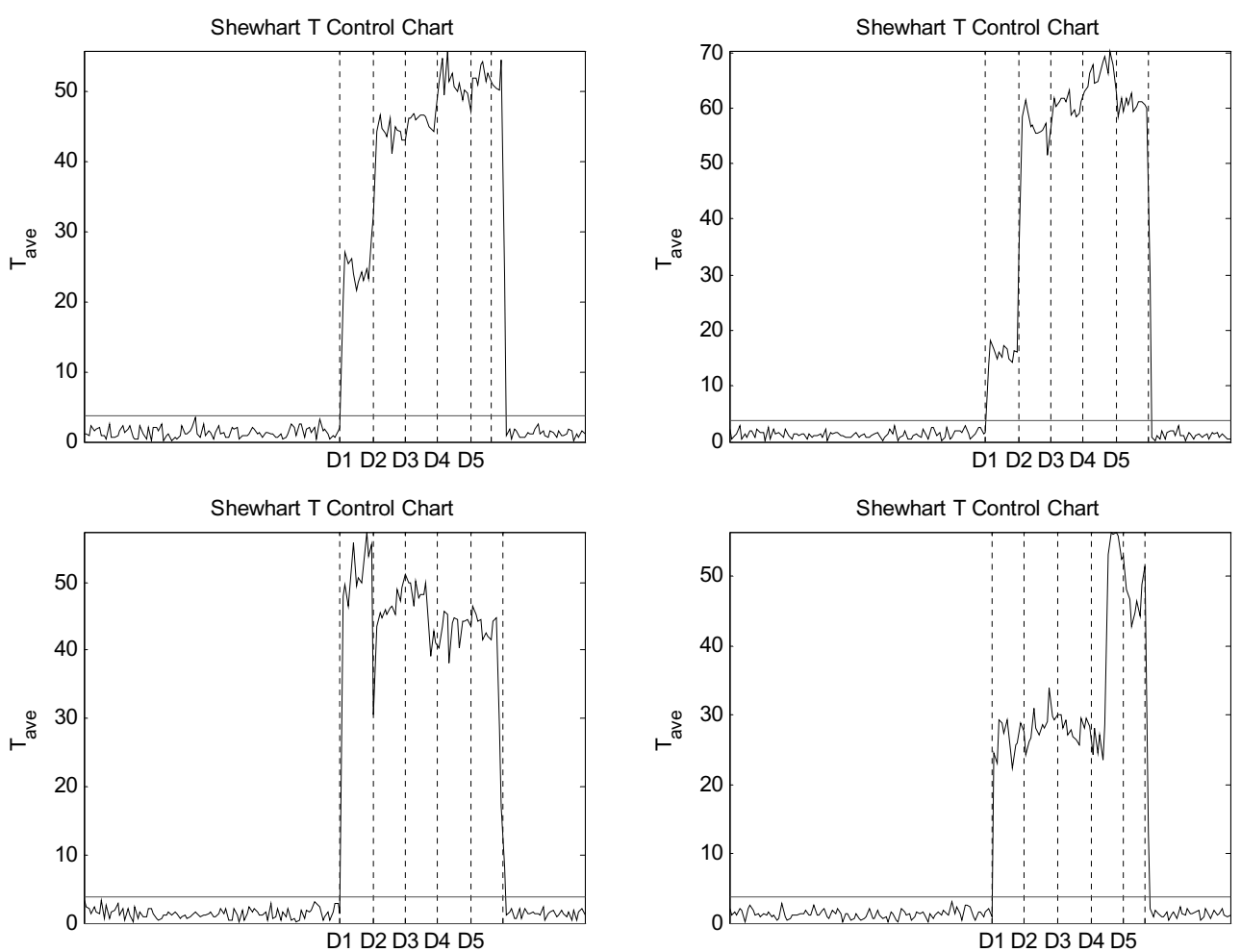

Fig. 9. Control charts for complex transmissibilities. Top left: damage location 1, 32 spectral lines. Top right: damage location 1, 64 spectral lines. Bottom left: damage location 2, 64 spectral lines. Bottom right: damage location 3, 64 spectral lines.

the proposed approach with factor analysis and followed by the principal component analysis, good results were obtained. The most sensitive results were obtained with complex transmissibilities having 64 spectral lines. These features showed also no false indications of damage.

\section{Conclusion}

The condition of a vehicle crane structure was monitored using multichannel measurements. No mathematical model of the structure was used. Damage detection was based on features extracted from the vibration measurements. Training data were first collected from the healthy structure. The performance of two different features to detect 
damage was studied: AR parameters and transmissibilities. The operational variations were first removed from the features using factor analysis. After this data normalization, the effectiveness of different features could be compared. Both features correctly detected all damage cases. Transmissibilities proved to be more sensitive to the introduced damage than the AR coefficients. The AR model may not correctly represent the dynamics of structures and a more appropriate approach might be the ARMA model. Complex transmissibilities with more features than the training samples showed the most sensitive detection capability with no false indications of damage. It should be noted, however, that the training data must include all operational variations and consequently the collection of the training data should be scheduled for a long time period. The conclusions were drawn from artificial damage with added masses. Similar study should also be made for more realistic damage scenarios with stiffness degradation. The feature extraction, operational variability elimination, and damage detection proposed in this paper can be easily automated, increasing the applicability of this research.

\section{Acknowledgements}

This research was performed in a MASINA technology program of the Finnish Funding Agency for Technology and Innovation (TEKES). The first author is also a partner in the S3HM project (www.s3hm.be) under the coordination of the ESF EUROCORES S3T program.

\section{References}

[1] S.W. Doebling, C.R. Farrar, M.B. Prime and D.W. Shevitz, Damage identification and health monitoring of structural and mechanical systems from changes in their vibration characteristics: A literature review. Los Alamos National Laboratory Report LA-13070-MS, 1996.

[2] H. Sohn, J.J. Czarnecki and C.R. Farrar, Structural health monitoring using statistical process control, Journal of Structural Engineering 126(11) (2000), 1356-1363.

[3] J. Kullaa, Damage detection under a varying environment using the missing data concept. Proceedings of the 5th International Workshop on Structural Health Monitoring. Fu-Kuo Chang (ed.). Stanford, CA. September 12-14, 2005. Stanford University. DEStech Publications. $565-573$.

[4] J. Kullaa, Structural health monitoring of a crane in variable configurations. Proceedings of ISMA2004, International Conference on Noise and Vibration Engineering, P. Sas and M. De Munck, eds, Leuven, Belgium, September 20-22, 2004, 457-469.

[5] K. Worden, G. Manson and N.R.J. Fieller, Damage detection using outlier analysis, Journal of Sound and Vibration 229(3) (1999), 647-667.

[6] R.P.C. Sampaio, N.M.M. Maia, J.M.M. Silva and A.M.R. Ribeiro, On the use of transmissibility for damage detection and location. Proc. of the European COST F3 Conference on System Identification \& Structural Health Monitoring, Madrid, June 7-9, 2000. Universidad Politécnica de Madrid, Spain. 363-376.

[7] Z. Sun and C.C. Chang, A wavelet packet based method for structural damage assessment. Proceedings of the Third World Conference on Structural Control, Volume 2, April 7-12, 2002, Como, Italy. International Association for Structural Control. Wiley. 3-8.

[8] A. Deraemaeker, E. Reynders, G. De Roeck and J. Kullaa, Vibration based SHM: comparison of the performance of modal features vs features extracted from spatial filters under changing environmental conditions. Proceedings of ISMA2006, International Conference on Noise and Vibration Engineering, P. Sas and M. De Munck, eds, Leuven, Belgium, September 18-20, 2006. 849-863.

[9] S.M. Kay, Modern Spectral Estimation: Theory and Application, Englewood Cliffs, NJ. Prentice-Hall, 1988,543 pages.

[10] D.C. Montgomery, Introduction to Statistical Quality Control, 3rd edition. New York. John Wiley \& Sons, 1997,728 pages.

[11] S. Sharma, Applied Multivariate Techniques, New York. John Wiley \& Sons, 1996, 493 pages.

[12] K.V. Mardia, J.T. Kent and J.M. Bibby, Multivariate Analysis, London. Academic Press, 1979, 521 pages. 

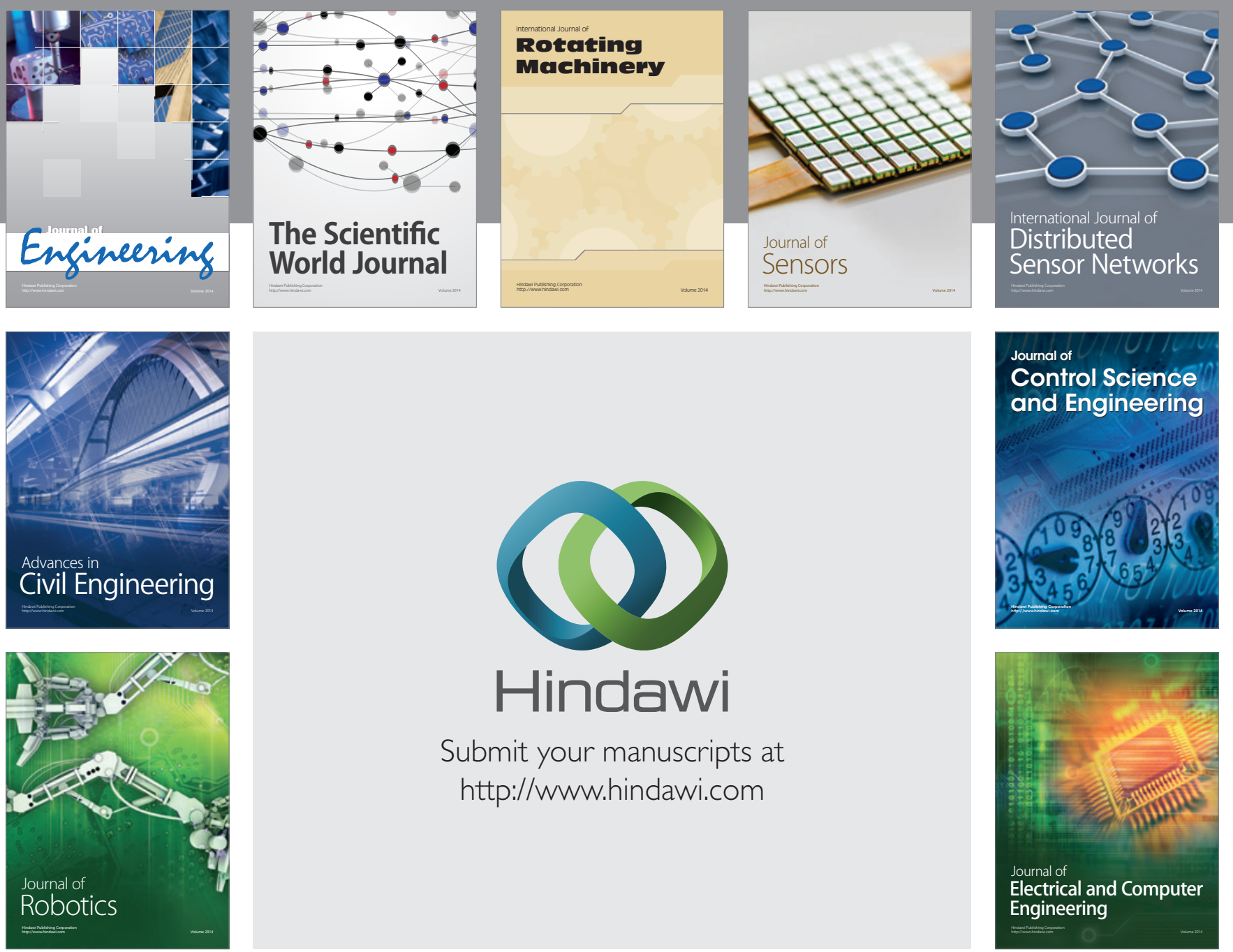

Submit your manuscripts at

http://www.hindawi.com
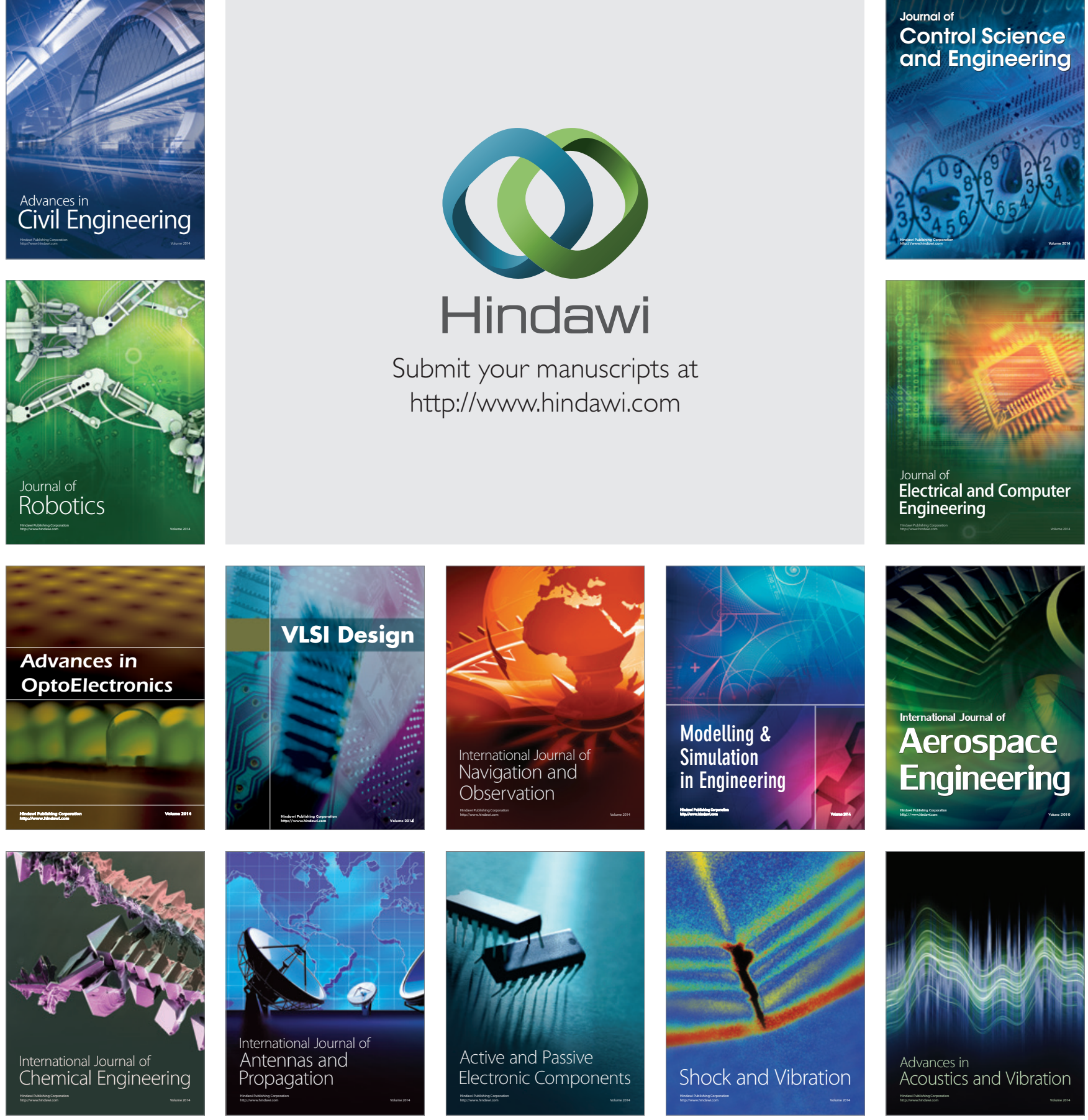